\title{
An Improved DV-Hop Localization Algorithm for Wireless Sensor Networks
}

\author{
Hongyang Chen ${ }^{1}$, Kaoru Sezaki ${ }^{2}$, Ping Deng ${ }^{3}$, Hing Cheung So ${ }^{4}$ \\ ${ }^{1}$ Department of Information and Communication Engineering, The University of Tokyo, Tokyo, \\ 153-8505 Japan. \\ ${ }^{2}$ Institute of Industrial Science, The University of Tokyo, Tokyo, 153-8505 Japan. \\ ${ }^{3}$ School of Information Science \& Technology, Southwest Jiaotong University, Chengdu, China \\ ${ }^{4}$ Department of Electronic Engineering, City University of Hong Kong, Hong Kong, China \\ Email: hongyang@mcl.iis.u-tokyo.ac.jp, sezaki@iis.u-tokyo.ac.jp,pdeng@home.swjtu.edu.cn, \\ ithcso@cityu.edu.hk
}

\begin{abstract}
Aiming at the positioning problem of wireless sensor network node location, an improved DV-Hop positioning algorithm is proposed in this paper, together with its basic principle and realization issues. The proposed method can improve location accuracy without increasing hardware cost for sensor node. Simulation results show that it has good positioning accuracy and coverage. The influences of anchor nodes on the DV-hop algorithm are also explored in the paper.
\end{abstract}

\section{INTRODUCTION}

With the development of sensor techniques, low-power electronic and radio techniques, low-power and inexpensive wireless sensors have been put into application, then the wireless sensor networks (WSNs) have appeared. WSNs can be applied to many areas, such as military affairs, commerce, medical care, environmental monitoring, and have become a new research focus in computer and communication fields. Many applications of WSNs are based on sensor self-positioning, such as battlefield surveillance, environment monitoring, indoor user tracking and others, which depend on knowing the location of sensor nodes. Because of the constraint in size, power, and cost of sensor nodes, the investigation of efficient location algorithms which satisfy the basic accuracy requirement for WSNs meets new challenges[1][2].

Many localization algorithms for sensor networks have been proposed to provide per-node location information. Based on the type of knowledge used in localization, we divide these localization protocols into two categories: range-based and range-free. Range-based protocols use absolute point-to-point distance or angle information to calculate the location between neighboring sensors. The second class of methods, range-free approach, employs to find the distances from the non-anchor nodes to the anchor nodes. Several ranging techniques are possible for range measurement, such as angle-of-arrival (AOA) [9], received signal strength indicator (RSSI)[13], time-of-arrival (TOA) [3] or time-difference-of-arrival (TDOA)[11]. Because of the hardware limitations of WSNs devices, solutions in range-free localization are being pursued as a cost-effective alternative to more expensive range-based approaches [4]. Because of the advantages on power and cost on sensor node, this paper focuses the investigation on the range-free algorithms for WSNs [14][15].

In this paper, we present an improved DV-Hop algorithm based on DV-Hop. The proposed method can improve location accuracy and coverage without increasing hardware cost of sensor node. Simulation results show that the performance of this algorithm is superior to the original DV-Hop algorithm. Compared with DV-Hop, it is more available for WSNs.

This paper makes three major contributions to the localization problem in WSNs. First, we present a practical, fast and easy-to-use localization scheme with relatively high accuracy and low cost for WSNs. Second, the proposed algorithm improves location accuracy and coverage than the DV-Hop algorithm. Third, we explored the influence of anchor nodes on localization performance of the DV-Hop algorithm.

The rest of this paper is organized as follows. Section II gives a survey of the related works. Section III presents the derivation of the proposed improved DV-Hop algorithm. In Section IV, simulation results are shown and localization performances are discussed. Finally, we present our conclusions in Section V.

\section{RELATED WORK}

In this section, we review research most relevant to our work. Many works in the literature propose schemes and methods for localization in WSNs for many purposes. Since each was developed to fulfill a different goal, they vary widely in many parameters including accuracy, cost, size, configurability, security, and reliability [5][6]. One example is Microsoft's RADAR [7] which uses existing 802.11 networks. Other recent research in ad hoc localization includes GPS, Active Bats [18], SpotON [8], Calamari [10].

In the range-free localization algorithms, Niculescu et al [2]. propose the DV-Hop localization scheme, which is similar to the traditional routing schemes based on distance vector. In their DV-Hop scheme, the node firstly counts the minimum hop 
number from the anchor node and then computes the distance between the node and anchor node by multiplying minimum hop number and average distance of each hop. At last, the node estimates its position through triangulation algorithm or maximum likelihood estimators (MLE). Nagpal et al. [4] improve the node localization accuracy by experimental model.

He et al. propose an approximate point-in triangulation test (APIT) algorithm in [4]. Using three anchors, APIT employs a area-based method to estimate node position. The effect of localization accuracy on applications also has been studied in [4].

Amorphous [17] algorithm is similar to the DV-Hop, but it assumes to know the network density in advance, and uses offline hop-distance estimations. It proposed to generate a rleatively accurate coordinate system on distributed processors via local information. Triangulation is also used to estimate a node's location.

Centroid algorithm [10] is a simple range-free localization algorithm. The node receives signals of landmarks in its communication area and makes its coordinates as the centroid of these landmarks. Additional devices of localization are not required in this algorithm. Thereby, the hardware of nodes can be simple. At the same time, its precision is comparatively low.

Centroid, DV-Hop, Amorphous, APIT are all distributed algorithms. They are characterized by simple computing, low traffic and scalable ability.

Range-free localization schemes are little affected by environmental factor, and additional range modules are not needed. These characteristics make them suitable to WSNs of simple node, low cost and large scale.

\section{IMPROVED DV-HOP LOCATION SCHEME}

Niculescu and Nath [2] have proposed the DV-Hop, which is a distributed, hop by hop positioning algorithm. The algorithm implementation is comprised of three steps. First, it employs a classical distance vector exchange so that all nodes in the network get distances, in hops, to the landmarks. And then, it estimates an average size for one hop, which is then deployed as a correction to the entire network. Finally, unknown nodes compute their location by trilateration [16].

\subsection{DV-Hop Algorithm}

In the first step, each anchor node broadcasts a beacon to be flooded throughout the network containing the anchors location with a hop-count value initialized to one. Each receiving node maintains the minimum hop-count value per anchor of all beacons it receives. Beacons with higher hop-count values to a particular anchor are defined as stale information and will be ignored. Then those not stale beacons are flooded outward with hop-count values incremented at every intermediate hop. Through this mechanism, all nodes in the network get the minimal hop-count to every anchor node.

In the second step, once an anchor gets hop-count value to other anchors, it estimates an average size for one hop, which is then flooded to the entire network. After receiving hop-size, blindfolded nodes multiply the hop-size by the hop-count value to derive the physical distance to the anchor. The average hop-size is estimated by anchor i using the following formula:

$$
\text { HopSize }_{i}=\frac{\sum_{j \neq i} \sqrt{\left(x_{i}-x_{j}\right)^{2}+\left(y_{i}-y_{j}\right)^{2}}}{\sum_{j \neq i} h_{i j}}
$$

where $\left(x_{i}, y_{i}\right),\left(x_{j}, y_{j}\right)$ are coordinates of anchor $i$ and anchor $j, h_{i j}$ is the hops between beacon $i$ and beacon $j$.

Each anchor node broadcasts its hop-size to network using controlled flooding. Unknown nodes receive hop-size information, and save the first one. At the same time, they transmit the hop-size to their neighbor nodes. This scheme could assure that the most nodes receive the hop-size from beacon node who has the least hops between them. In the end of this step, unknown nodes compute the distance to the beacon nodes based hop-length and hops to the beacon nodes.

Let $(x, y)$ be the unknown node $\mathrm{D}$ location and $\left(x_{i}\right.$, $\left.y_{i}\right)$ the known location of the i'th anchor node receiver. Let's say the i'th anchor node distance to unknown nodes is di, well then, there is the following formula:

$$
\left\{\begin{array}{c}
\left(x-x_{1}\right)^{2}+\left(y-y_{1}\right)^{2}=d_{1}^{2} \\
\left(x-x_{2}\right)^{2}+\left(y-y_{2}\right)^{2}=d_{2}^{2} \\
\vdots \\
\left(x-x_{i}\right)^{2}+\left(y-y_{i}\right)^{2}=d_{i}^{2}
\end{array}\right.
$$

the coordinates of $\mathrm{D}$ is computed by the following formula:

$$
\begin{gathered}
A=-2 \times\left[\begin{array}{cc}
x_{1}-x_{n} & y_{1}-y_{n} \\
x_{2}-x_{n} & y_{2}-y_{n} \\
\vdots & \vdots \\
x_{n-1}-x_{n} & y_{n-1}-y_{n}
\end{array}\right] \\
B=\left[\begin{array}{c}
d_{1}^{2}-d_{n}^{2}-x_{1}^{2}+x_{n}^{2}-y_{1}^{2}+y_{n}^{2} \\
d_{2}^{2}-d_{n}^{2}-x_{2}^{2}+x_{n}^{2}-y_{2}^{2}+y_{n}^{2} \\
\vdots \\
d_{n-1}^{2}-d_{n}^{2}-x_{n-1}^{2}+x_{n}^{2}-y_{n-1}^{2}+y_{n}^{2}
\end{array}\right] \\
P=\left[\begin{array}{c}
x \\
y
\end{array}\right] \\
P=\left(A^{T} A\right)^{-1} A^{T} B .
\end{gathered}
$$

\subsection{Improved DV-Hop Algorithm}

In this subsection, we improve DV-Hop algorithm focus on step 2 and step 3 .

In step 2, after obtained the hop-size, anchor node broadcasts its hop-size to network as a correction. The format of the package is $\{\mathrm{Id}$, Hop-Sizei\}, including the identifier id, an average size for one hop Hop-Sizei. Once a node gets the 
package, it adds the information to a table and also broadcasts it to neighbor nodes. The package for iterative ID will be dropped. After the step 1 for broadcast, all of the nodes get the Hop-Sizei , which calculated by the anchor nodes in the first step of DV-Hop algorithm. We average whole of the hop-size of different anchor nodes using the following formula:

$$
\text { HopSize }_{\text {ave }}=\frac{\sum \text { HopSize }_{i}}{n}
$$

where $n$ is the number of anchor nodes, HopSize is obtained using (3-1). In the end of this step, unknown nodes compute the distance to the beacon nodes based hop-length and hops to the beacon nodes by the formula:

$$
d_{i}=\text { hops } \times \text { HopSize }_{\text {ave }}
$$

In step 3, a general model for two dimensional (2-D) position location estimation of a source using $M$ anchor nodes is developed. Let $(x, y)$ be the source node location and $\left(x_{i}\right.$ , $\left.y_{i}\right)$ be the known location of the i'th anchor node receiver. Denote the distance between the unknown node and anchor node $\mathrm{i}$ by $d_{i}$. It is clear that

$$
d_{i}=\sqrt{\left(X_{i}-x\right)^{2}+\left(Y_{i}-y\right)^{2}} \text {. }
$$

In DV-Hop algorithm, the estimated physical distances are used together with the anchor positions to perform a triangulation in order to get the final localization results. In our improved DV-Hop localization system, we will not adopt traditional Triangulation algorithm but use 2-D Hyperbolic location algorithm [9][12].

So we propose to use a least square method to give a good estimation of such a starting point [12].

By the definition of (3-8), we have the following expression:

$X_{i}^{2}+Y_{i}^{2}-2 X_{i} x-2 Y_{i} y+x^{2}+y^{2}=d_{i}^{2} \Rightarrow$

$d_{i}^{2}-E_{i}=-2 X_{i} x-2 Y_{i} y+K$

where $E_{i}=X_{i}^{2}+Y_{i}^{2}, K=x^{2}+y^{2}$.

$$
\text { Let } Z_{c}=[x, y, K]^{T} \text {, }
$$

$$
G_{c}=\left[\begin{array}{ccc}
-2 X_{1} & -2 Y_{1} & 1 \\
-2 X_{2} & -2 Y_{2} & 1 \\
\vdots & \vdots & \vdots \\
-2 X_{i} & -2 Y_{i} & 1
\end{array}\right] \text {, }
$$

(3-11)

and

$$
h_{c}=\left[\begin{array}{c}
d_{1}^{2}-E_{1} \\
d_{2}^{2}-E_{2} \\
\vdots \\
d_{i}^{2}-E_{i}
\end{array}\right],
$$

by (3-9), we can have

$$
G_{c} Z_{c}=h_{c}
$$

Using Least Square (LS) algorithm [12], from (3-13) we can get

$$
Z_{c}=\left(G_{c}^{T} G_{c}\right)^{-1} G_{c}^{T} h_{c}
$$

Then, the coordinates of the unknown node, $(x, y)$ is expressed as:

$$
\left\{\begin{array}{l}
x=Z_{c}(1), \\
y=Z_{c}(2) .
\end{array}\right.
$$

\section{Simulation Results and Performance analysis}

In this section, simulation results are presented and analyzed. We simulated the DV-Hop and our proposed algorithm to evaluate location performance. The experiment region is a square area with the fixed size of $50 \times 50 \mathrm{~m} 2$ and the radio range of sensor nodes $(\mathrm{R})$ is set to 10 meter. As shown in Fig.1, We deploy 100 sensor nodes randomly in a two-dimensional space.

The simulation results presented as flow figures:

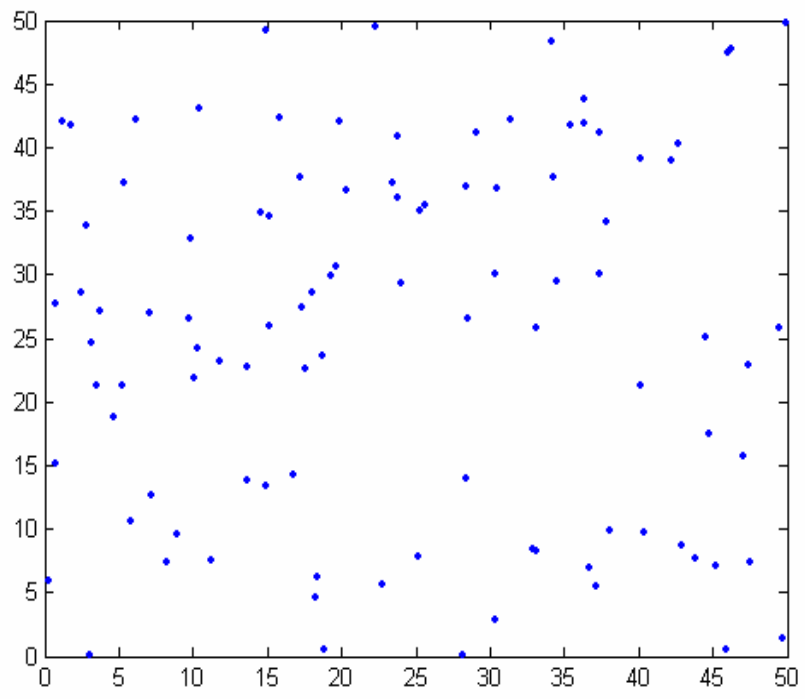

Fig.1. Nodes distribution 


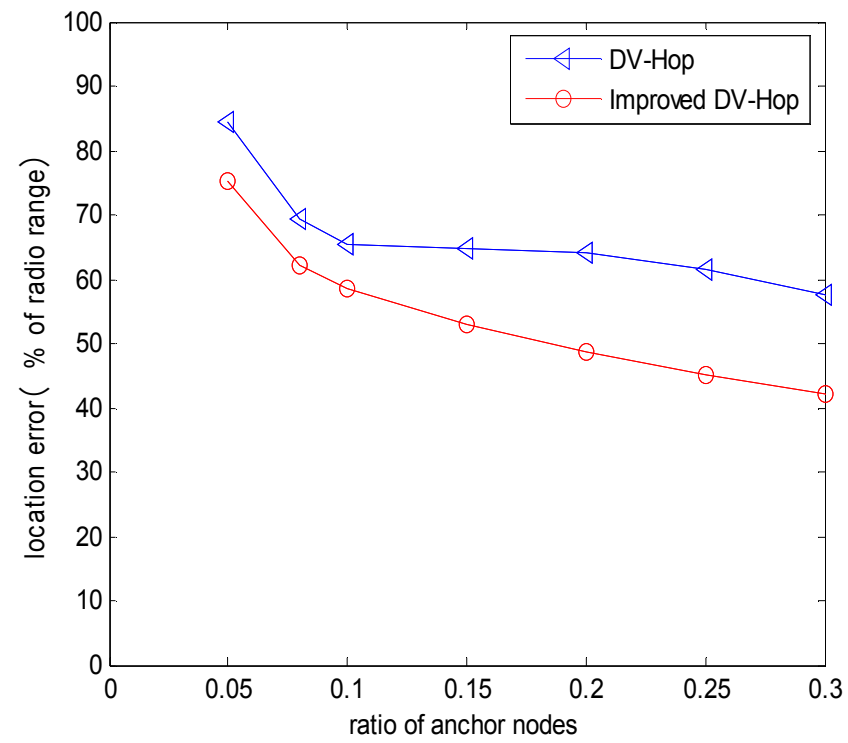

Fig.2. Location error

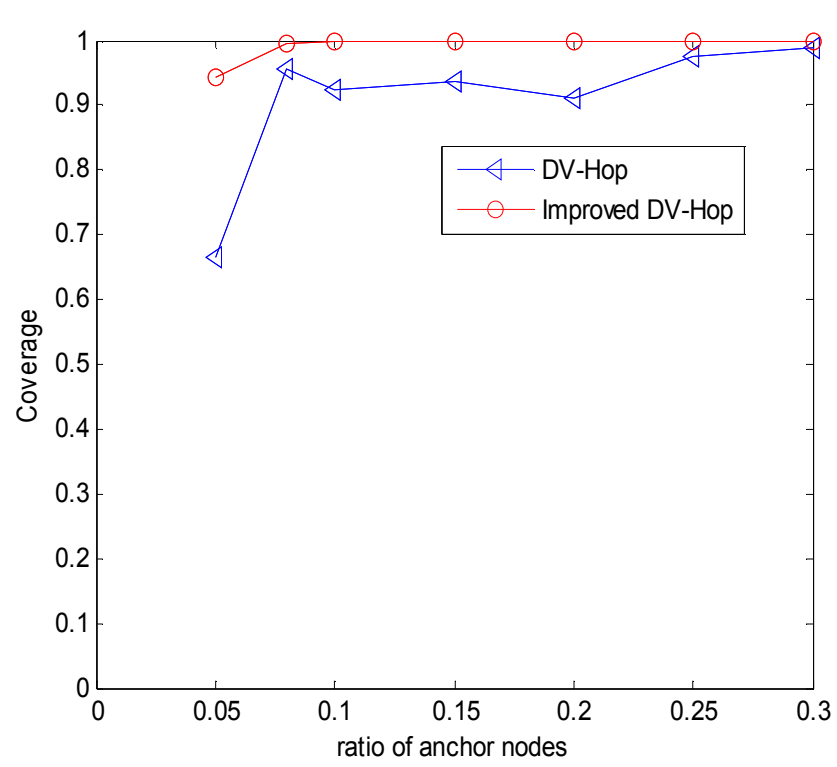

Fig.3. Location coverage

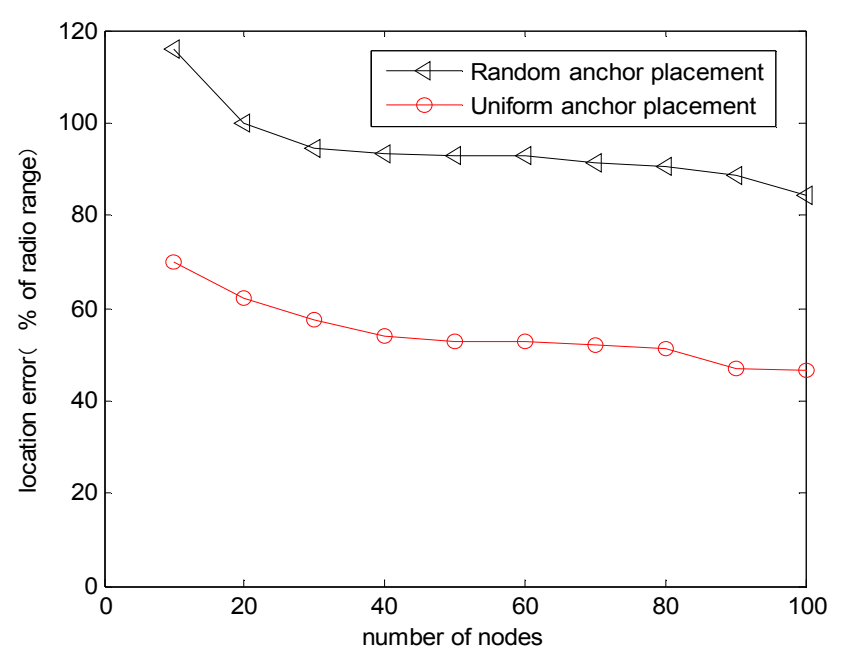

Fig.4. Location error

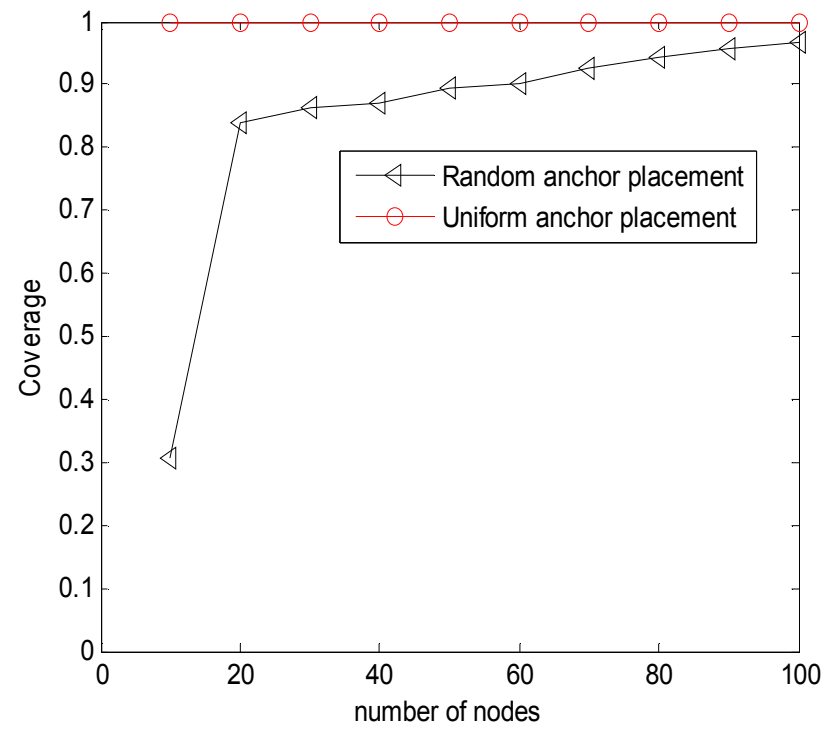

Fig.5. Location coverage

As can be seen from the simulation results of Fig.2 and Fig.3, our improved DV-Hop algorithm achieve better performance than the DV-Hop algorithm. The location error decreases as ratio of anchor nodes increase. For the same ratio of anchor nodes, position error is smaller when our improved DV-Hop scheme applied in same WSNs environment than the DV-Hop algorithm. For example, with 5 anchor nodes (5\%), Improved DV-Hop has an average error of about $75 \% \mathrm{R}$, where as the DV-Hop has an average error of about $84 \%$ R. From Fig.4, we will sure the location coverage improved via using our improved DV-Hop algorithm. For example, with 10 anchor nodes $(10 \%)$, Improved DV-Hop algorithm reaches location coverage about $100 \%$.

The placement of anchor nodes will affect the DV-Hop algorithm. From Fig.4 and Fig. 5, it can be stated that important improvements in the positioning accuracy and location coverage are obtained when uniform anchor placement instead of random anchor placement. Simulation results show that the more regularly anchor nodes are placed, the lower the error.

The performance of our Improved DV-Hop scheme exceeds the original DV-Hop location algorithm from our simulation results.

\section{CONCLUSION}

We present a new method, Improved DV-Hop, that improves the basic DV-Hop algorithm significantly. It is shown in the simulation results that the proposed algorithm can improve location accuracy and coverage than the original DV-Hop algorithm. As shown in the simulation results, it can be stated that the approach is effective and obviously has good application foreground. We also explored the influence of anchor nodes on the DV-hop algorithm. Not only the placement of anchor nodes, but also the quantity of anchor nodes could affect the localization average error and location coverage of the algorithm. Simulation results show that the more regularly 
beacons are placed, the lower the error and higher location coverage. In the application scenario, we can place anchor nodes by manual work, location performance can be improved then.

\section{REFERENCES}

[1] A.I.F., W.Su, S. Y, and C. E, "A Survey on Sensor Networks," in Proc. IEEE Communications Magazine, vol.40, pp.102-113, Aug. 2002.

[2] D. Niculescu, B. Nath. "Ad Hoc Positioning System (APS)," Proc. of the IEEE GLOBECOM 2001, San Antonio, pp. 2926-2931, 2001.

[3] G.L, E.D, "Robust range estimation using acoustic and $n$ multimodal sensing," IEEE International Conference on Intelligent Robots and Systems, vol 3, pp. 1312-1320, 2001.

[4] T. He, C. Huang, B. M. Blum, J. A. Stankovic, T.Abdelzaher. "Range-Free Localization Schemes for Large Scale Sensor Networks," Proc. of the ACM MobiCom 2003, San Diego, pp. 81-95, 2003.

[5] S.Meguerdichian, F.Koushanfar, M.Potkonjak, and M.B. "Srivastava,Coverage Problems in Wireless Ad-hoc Sensor Neworks," IEEE INFOCOM2001, Ankorange, Alaska, pp. 1380-1387,April 2001.

[6] N.Bulusu,J.Heidemann,J.Estrin, "Adaptive beacon placement," International Conference on Distributed Computing Systems, Phoenix, Arizona, pp.489-498,April,2001.

[7] P.Bahl and V.N. Padmanabhan. "RADAR: An in-building RF-based user location and tracking system," in IEEE INFOCOM2000, vol.2, pp.775-784, March 2000.

[8] J.Hightower, C.Vakili, G.Borriello and R.Want, "Design and calibration of the SPOTON ad-hoc location sensing system," University of Washington, Seattle, WA, August 2001.

[9] T. D. J. "Statistical theory of passive location systems," IEEE Trans. on AES. vol.20, no.2, pp. 183-198, Mar.1984.

[10] C.S, H. M, H.J, "Gps-Free positioning in mobile Ad Hoc networks," in Proc. of Hawaii Int'l. Conf. System Sciences, pp.3481-3490, 2001.

[11] X. Cheng, T. A, G. Xue, D. Chen, "TPS: a time-based positioning scheme for outdoor wireless sensor networks," IEEE INFOCOM2004, Hong Kong, China. pp. 2685-2696, March,2004.

[12] Y. T. Chan and K. C. Ho, "A simple and efficient estimator for hyperbolic location," IEEE Transactions on Signal Processing, vol. 42, pp. 1905-1915, August 1994.

[13] T.S.Rappapport, Wireless Communications: Principles and Practice. Prentice Hall: New Jersey, pp.50-143,1996.

[14] A. Savvides, C.C. Han, and M. Srivastava, "Dynamic fine-grained localization in ad-hoc networks of sensors," in Proceeding of the 7 th ACM International Conference on Mobile Computing and Networking (MOBICOM), Rome, Italy , pp.166-179,July 2001.

[15] J.Chen, K. Yao, and R. Hudson, "Source Localization and Beamforming," IEEE Signal Processing Magazine, vol. 19, pp. 30-39, Mar. 2002.

[16] L.Doherty, K.Pister, L.E.Ghaoui, "Convex position estimation in wireless sensor networks," in IEEE INFOCOM 2001, Anchorage, AK,2001.

[17] R. Nagpal, "Organizing a global coordinate system from local information on an amorphous computer," A.I. Memo 1666, MIT A.I. Laboratory, Aug. 1999.

[18] A. Harter and A. Hopper, "A distributed location system for the active office. ," IEEE Network, Vol. 8, No. 1, Jan. 1994 